\title{
Analysis of the Clinicopathological Characteristics of Gastric Cancer in Extremely Old Patients
}

\author{
II Woong Sohn, MD \\ Da Hyun Jung, MD² \\ Jie-Hyun Kim, MD, PhD² \\ Hyun Soo Chung, MD \\ Jun Chul Park, MD' \\ Sung Kwan Shin, MD, PhD' \\ Sang Kil Lee, MD, $P h D^{1}$ \\ Yong Chan Lee, MD, $\mathrm{PhD}^{1}$
}

${ }^{1}$ Department of Internal Medicine, Yonsei University College of Medicine, Seoul, ${ }^{2}$ Department of Internal Medicine, Gangnam Severance Hospital, Yonsei University College of Medicine, Seoul, Korea

Correspondence: Da Hyun Jung, MD

Department of Internal Medicine,

Gangnam Severance Hospital,

Yonsei University College of Medicine,

211 Eonju-ro, Gangnam-gu, Seoul 06273, Korea

Tel: 82-2-2019-3305

Fax: 82-2-3463-3882

E-mail: leah1004@yuhs.ac

Received April 18, 2016

Accepted June 7, 2016

Published Online June 27, 2016

\section{Purpose}

Gastric cancer is the third-leading cause of cancer-related death in Korea. As the Korean population is ageing, the number of extremely old patients with this disease is increasing. This study examined the clinicopathological characteristics of gastric cancer in extremely old (over 85 years) patients who received treatment or conservative observations and compared the treatment outcomes according to the treatment modality.

\section{Materials and Methods}

A total of 170 patients over 85 years of age were diagnosed with gastric cancer. Of these, 81 underwent treatment for gastric cancer and 89 received conservative observations. The clinicopathological characteristics of the treatment and conservative groups were compared.

\section{Results}

The mean age of the patients was 86.5 years. The conservative group included significantly more patients with older ages, macroscopically advanced cancer and upper-middle located cancer. The overall survival rate of the treatment group was significantly higher than that of the conservative group. The disease-specific mortality rate was significantly lower in the treatment group than in the conservative group. Multivariate analysis revealed the clinical course, alarm sign, and macroscopic classification to be independent prognosis factors.

\section{Conclusion}

By itself, the chronological age should not be used as a strategy to determine whether treatment will be administered for gastric cancer. Patients who have early gastric cancer or lowerrisk preexisting comorbidities should not be discouraged from treatment, even if they are older than 85 years.

\section{Introduction}

Following thyroid cancer, gastric cancer (GC) is the second most common cancer in Korea [1]. Recently, as the Korean society is aging, there has been an increasing number of elderly patients diagnosed with GC. Despite this, there is little data available on the clinicopathological characteristics of GC in elderly patients, particularly in those who are very elderly. In Korea, the life expectancy is more than 80 years and the
Key words

Oldest old, Aged, Stomach neoplasms, Therapy most common cause of death is cancer [2]. According to the statistics released by Statistics Korea, approximately $12 \%$ of cancer-related deaths have been caused by GC in recent years. Although the 5-year survival rate of GC has been increasing over the past two decades due to early detection by screening endoscopy [3], little is known about GC screening in the very elderly.

Endoscopic treatment is the accepted standard treatment for selected cases of GC. The safety of endoscopic treatment is comparable to surgery in elderly patients [4-8]. Endoscopic 
resections (ERs) are also performed on elderly patients with comorbid heart and lung diseases [9]. Therefore, the strategy of GC treatment varies in elderly patients with age-associated comorbidities. Age is a clinically important factor for determining the treatment modality [10], and clinicians are often cautious when managing elderly patients with GC because of their age-related performance status [11-13].

This study investigated the clinicopathological characteristics of GC in extremely old (over 85 years) patients who received treatment or conservative observations. In addition, the treatment outcomes were compared according to the treatment modality.

\section{Materials and Methods}

\section{Patients}

From June 1998 to July 2014, a total of 358 patients over 85 years old were diagnosed with GC at Severance Hospital (Seoul, Korea). For the present analysis, the following patients were excluded (1) those who had no subsequent follow-up visits after diagnosis, (2) those who had a significant comorbidity that could affect the mortality, and (3) those who had been treated at another hospital during the followup period. Of the 358 patients, 188 were excluded and the remaining 170 were enrolled. The patients were evaluated in terms of their general information, such as age, sex, and treatment method. The variables investigated also included the macroscopic classification, histological classification, comorbidity, postoperative mortality, and clinical outcome. As categorized by the treatment modality for GC, 81 patients underwent treatment (treatment group), such as an endoscopic resection, surgery, chemotherapy, or radiotherapy, and 89 patients received conservative observation (conservative group). The staging of the patients in the conservative group could not be evaluated accurately. Therefore, the patients were divided roughly into two groups: those with macroscopically early GCs (cancer confined to the mucosa or submucosa) and those with macroscopically advanced GC (cancer with invasion extending through the muscularis propria). The patients' medical records were reviewed retrospectively. The Institutional Review Board (IRB) of Severance Hospital approved this study (IRB approval number: 4-20140464).

\section{Statistical analysis}

The chi-square test and Fisher exact test were used to compare the clinicopathological characteristics between the treat- ment-modality groups. Follow-up data on all patients were obtained from the Korea National Health Insurance Service database. The median follow-up period was 17.8 months (range, 0.1 to 172.4 months). The survival curves were estimated using the Kaplan-Meier method. The accepted significant level was $p<0.05$. All statistical analyses were performed using SPSS ver. 20.0 for Windows (IBM Co., Armonk, NY).

\section{Results}

\section{Clinicopathological characteristics}

The sex or histologic type was similar in the two groups. On the other hand, older age, macroscopically advanced cancer, and upper-middle located cancer were significantly more common in the conservative group (Table 1). In the treatment group, 21 patients underwent ER, 48 patients underwent gastrectomy, and 12 patients underwent chemotherapy or radiotherapy. Of the 21 patients who received ER, 20 had lesions included in the absolute indication and one patient had lesions included in the expanded indication. Of the 48 patients who received surgery, 46 underwent a curative gastric resection (subtotal gastrectomy, $n=43$; total gastrectomy, $\mathrm{n}=3$ ) and two received palliative gastrectomy. The curative resection rate was 18/21 (85.7\%) for ER and 46/48 $(95.8 \%)$ for gastrectomy. In the conservative observation group, 38/89 patients $(42.7 \%)$ refused the recommended treatment.

In the overall study cohort, the most common concomitant disease was hypertension (32.9\%). Eighty-two patients $(48.2 \%)$ had one or more comorbid diseases and 37 patients $(21.8 \%)$ had more than two comorbid diseases.

One hundred forty-three patients $(84.1 \%)$ visited the hospital with symptoms and 27 patients $(15.9 \%)$ were diagnosed with GC during a health check-up. The most common initial presentation was abdominal pain, which was present in 69 patients $(40.6 \%)$. Thirty-seven patients $(21.8 \%)$ had alarm signs (weight loss, signs and symptoms of upper gastrointestinal bleeding, and anemia), and the presence of an alarm sign was significantly more common in the conservative observation group than in the treatment group. On the other hand, indigestion was significantly more common in the treatment group.

\section{Clinical outcomes}

A postoperative complication (bleeding) occurred in one patient who underwent a gastrectomy; this patient died three 
Table 1. Clinicopathological characteristics of the treatment and conservative observation groups

\begin{tabular}{|c|c|c|c|}
\hline Factor & $\begin{array}{l}\text { Treatment group } \\
\qquad(\mathrm{n}=\mathbf{8 1})\end{array}$ & $\begin{array}{l}\text { Conservative group } \\
\qquad(\mathrm{n}=89)\end{array}$ & p-value \\
\hline Age (yr) & $85.9 \pm 1.9$ & $87.1 \pm 2.6$ & \\
\hline Range & $85.0-94.0$ & $85.0-96.0$ & 0.003 \\
\hline \multicolumn{4}{|l|}{ Sex } \\
\hline Male & $58(71.6)$ & $53(59.6)$ & 0.109 \\
\hline Female & $23(28.4)$ & $36(40.4)$ & \\
\hline \multicolumn{4}{|l|}{ Macroscopic classification } \\
\hline Early & $34(42.0)$ & $12(13.5)$ & $<0.001$ \\
\hline Advanced & $47(58.0)$ & $77(86.5)$ & \\
\hline \multicolumn{4}{|l|}{ Histologic type } \\
\hline Differentiated & $55(67.9)$ & $58(65.2)$ & 0.747 \\
\hline Undifferentiated & $26(32.1)$ & $31(34.8)$ & \\
\hline \multicolumn{4}{|l|}{ Location } \\
\hline Upper & $5(6.2)$ & $22(24.7)$ & 0.001 \\
\hline Middle & $23(28.4)$ & $30(33.7)$ & \\
\hline Lower & $53(65.4)$ & $35(39.3)$ & \\
\hline Unknown & 0 & $2(2.2)$ & \\
\hline Tumor size (cm) & $4.63 \pm 3.42$ & $4.64 \pm 2.44$ & 0.981 \\
\hline \multicolumn{4}{|l|}{ Distant metastasis } \\
\hline Absence & $74(91.4)$ & $50(56.2)$ & $<0.001$ \\
\hline Presence & $7(8.6)$ & $25(28.1)$ & \\
\hline Unknown & 0 & $14(15.7)$ & \\
\hline \multicolumn{4}{|l|}{ Concomitant disease } \\
\hline None & $40(49.4)$ & $48(53.9)$ & 0.645 \\
\hline Hypertension & $28(34.6)$ & $28(31.5)$ & 0.744 \\
\hline Cardiovascular disease & $5(6.2)$ & $3(3.4)$ & 0.481 \\
\hline Diabetes mellitus & $13(16.0)$ & $11(12.4)$ & 0.516 \\
\hline Cerebral infarction & $13(16.0)$ & $3(3.4)$ & 0.007 \\
\hline Chronic pulmonary disease & $2(2.5)$ & $4(4.5)$ & 0.684 \\
\hline Chronic kidney disease & $1(1.2)$ & $2(2.2)$ & $>0.99$ \\
\hline Other malignancy & $3(3.7)$ & $7(7.9)$ & 0.335 \\
\hline \multicolumn{4}{|l|}{ Initial presentation } \\
\hline Abdominal pain & $30(37.0)$ & $39(43.8)$ & 0.435 \\
\hline Indigestion & $21(25.9)$ & $10(11.2)$ & 0.017 \\
\hline Hematemesis & $4(4.9)$ & $9(10.1)$ & 0.255 \\
\hline Melena & $2(2.5)$ & $8(9.0)$ & 0.103 \\
\hline Hematochezia & $2(2.5)$ & 0 & 0.226 \\
\hline Anemia & $1(1.2)$ & $7(7.9)$ & 0.068 \\
\hline General weakness & $1(1.2)$ & $5(5.6)$ & 0.215 \\
\hline Weight loss & $2(2.5)$ & $2(2.2)$ & $>0.99$ \\
\hline None & $18(22.2)$ & $9(10.1)$ & 0.037 \\
\hline
\end{tabular}

Values are presented as mean \pm standard deviation or number (\%) unless otherwise indicated.

days after surgery. No other procedure-related complications were observed in the patients who received ER. Recurrence developed in two of the patients who received ER. One patient had a local recurrence and the other patient developed liver metastasis. Eight of the patients who underwent surgery experienced a recurrence.

\section{Survival}

The overall survival rate in the treatment group was significantly higher than that in the conservative group (Fig. 1A). Furthermore, the disease-specific mortality was significantly lower in the treatment group than in the con- 
A
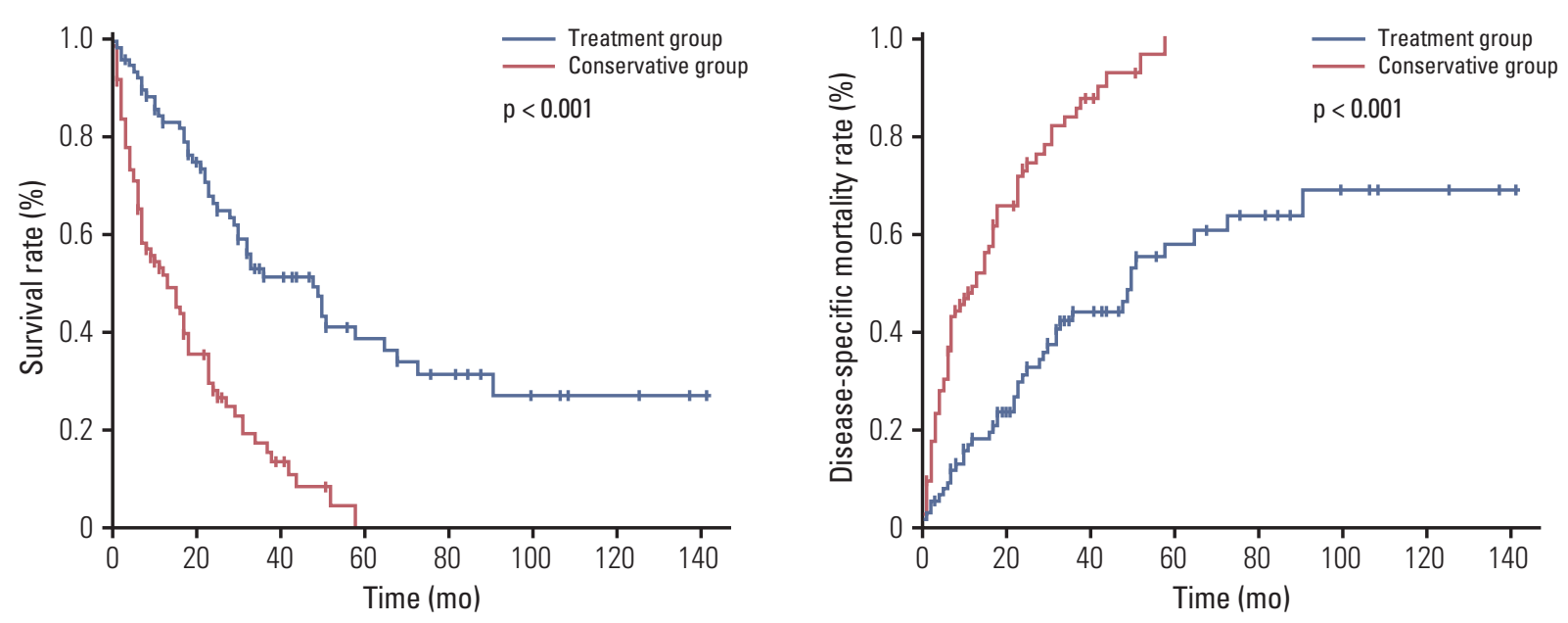

Fig. 1. (A) Overall survival rate of the treatment $(n=81)$ and conservative $(n=89)$ groups. (B) Disease-specific mortality of the treatment $(n=81)$ and conservative $(n=89)$ groups.

\section{A}
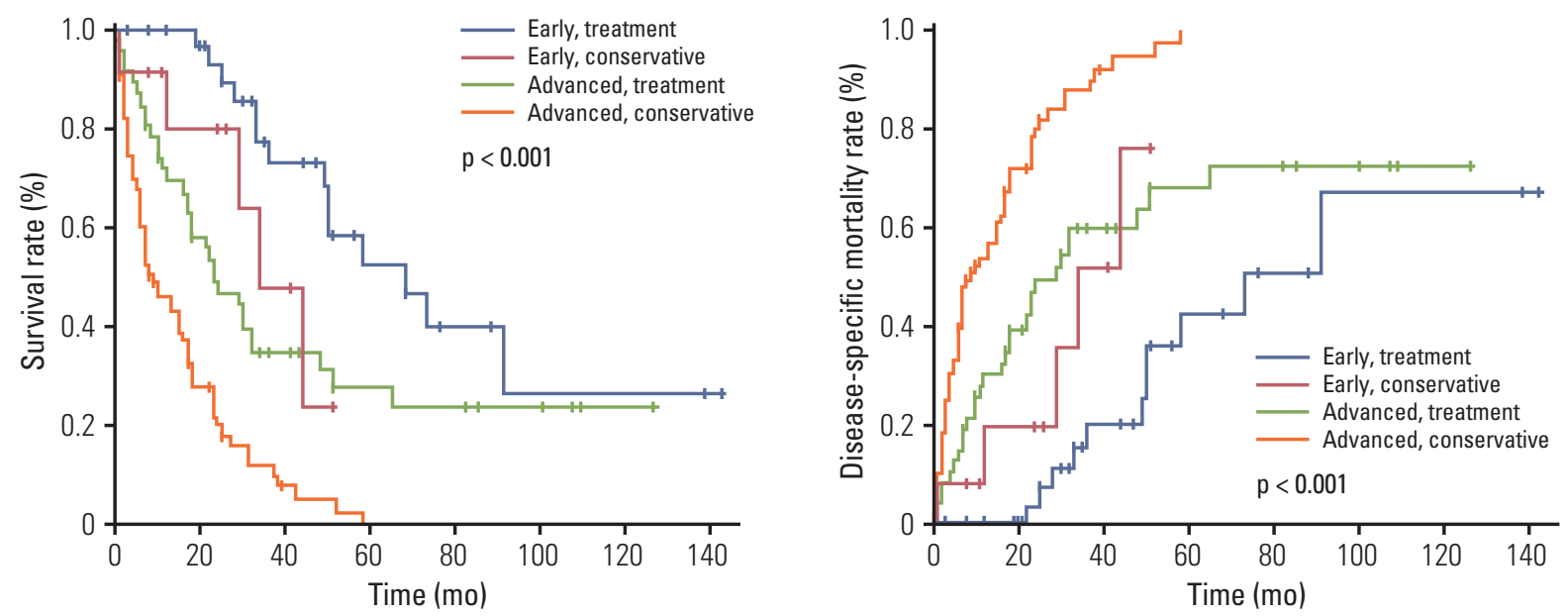

Fig. 2. The patients in the treatment group and the conservative group were divided into early gastric cancer (GC) and advanced GC subgroups (early and treatment group, $n=34$; early and conservative group, $n=12$; advanced and treatment group, $n=47$; and advanced and conservative group, $n=77$ ). (A) Overall survival rate with GC patients showing macroscopic classification and clinical course. (B) Disease-specific mortality rate with GC patients showing macroscopic classification and clinical course.

servative group (Fig. 1B). The 81 patients in the treatment group and the 89 patients in the conservative group were divided into early GC and advanced GC subgroups, as shown in Fig. 2. For the patients with advanced GC, the overall survival rate was significantly higher in the treatment group than in the conservative group. For patients with early
GC, the overall survival rate was also significantly higher in the treatment group than in the conservative group $(p<0.001)$ (Fig. 2A). Similarly, the disease-specific mortality in the treatment group was significantly lower than that in the conservative group, regardless whether early or advanced GC had been diagnosed $(p<0.001)$ (Fig. 2B). Sub- 
A
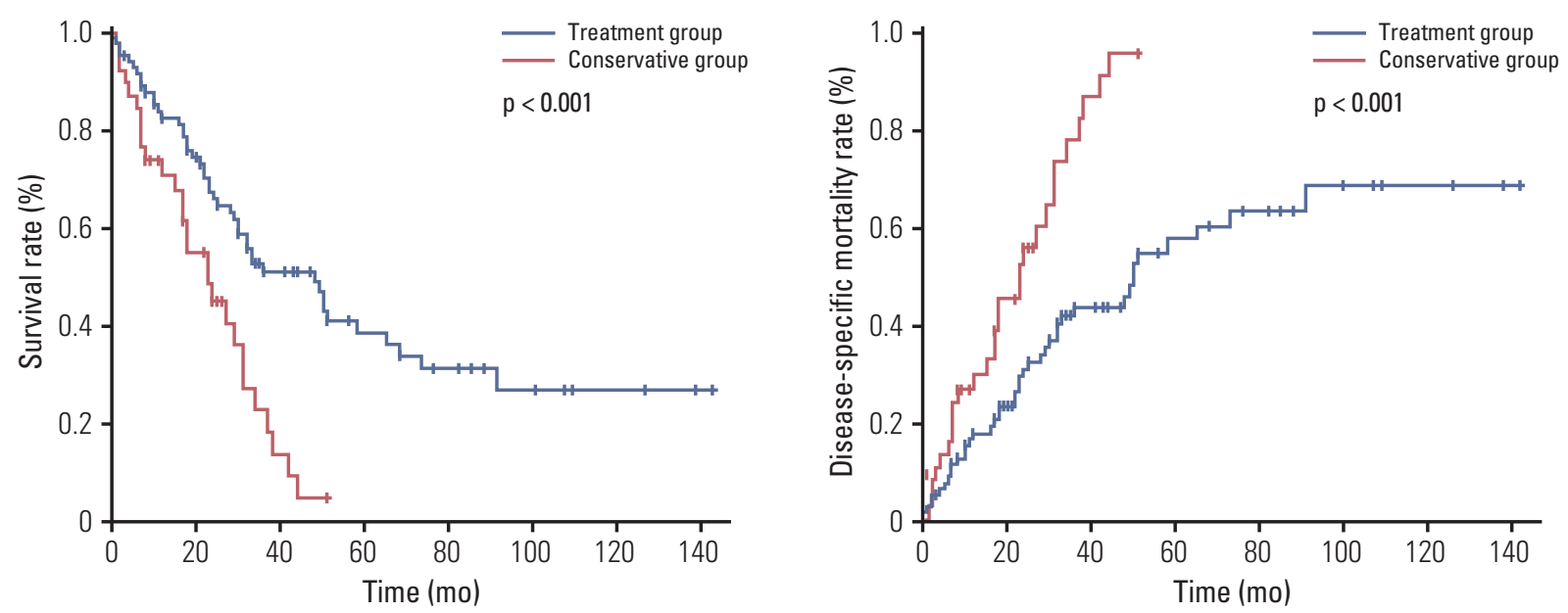

Fig. 3. (A) Overall survival rate of the treatment $(n=81)$ and conservative $(n=38)$ groups refused recommended treatment. (B) Disease-specific mortality of the treatment $(n=81)$ and conservative $(n=89)$ groups refused recommended treatment.

A
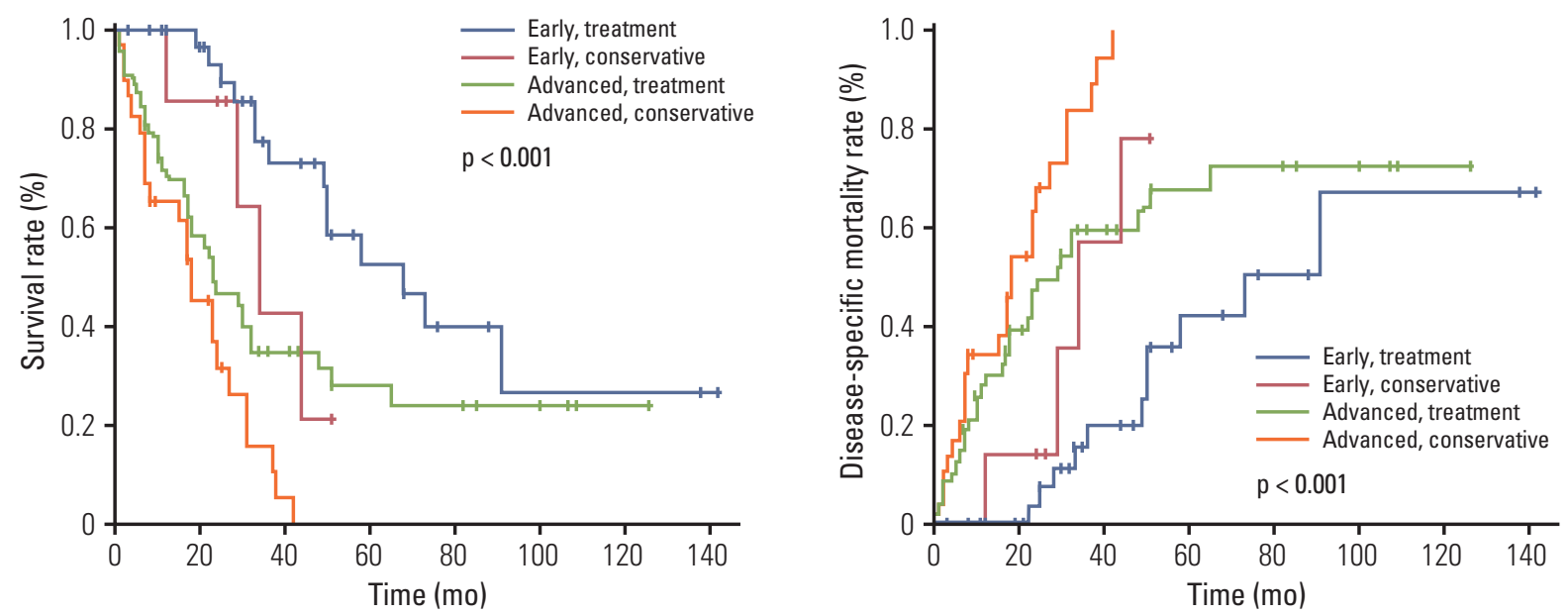

Fig. 4. The patients in the treatment group and the conservative group refused recommended treatment were divided into early gastric cancer (GC) and advanced GC subgroups (early and treatment group, $n=34$; early and conservative group, $n=9$; advanced and treatment group, $n=47$; and advanced and conservative group, $n=29$ ). (A) Overall survival rate with GC patients showing macroscopic classification and clinical course. (B) Disease-specific mortality rate with GC patients showing macroscopic classification and clinical course.

group analysis was performed on the overall survival and disease specific mortality between the conservative observation group who refused the recommended treatment and the treatment group. The overall survival rate of the treatment group was significantly higher than the conservative observation group who refused the recommended treatment and the disease specific mortality rate was significantly lower (Fig. 3). The patients in the treatment group and the conservative observation group who refused the recommended treatment were divided into early GC and advanced GC subgroups. The overall survival rate was significantly higher and the disease-specific mortality rate was significantly 
Table 2. Correlation between the prognostic factors and the survival rates of 170 gastric cancer patients

\begin{tabular}{|c|c|c|c|}
\hline Factor & No. & $\begin{array}{c}3-Y_{\mathbf{r}} \\
\text { survival rate }(\%)\end{array}$ & p-value \\
\hline \multicolumn{4}{|l|}{ Clinical course } \\
\hline Treatment group & 81 & 50.7 & $<0.001$ \\
\hline Conservative observation & 89 & 16.8 & \\
\hline \multicolumn{4}{|l|}{ Age (yr) } \\
\hline$<90$ & 150 & 35.0 & 0.027 \\
\hline$\geq 90$ & 20 & 26.4 & \\
\hline \multicolumn{4}{|l|}{ Sex } \\
\hline Male & 111 & 32.8 & 0.525 \\
\hline Female & 59 & 36.1 & \\
\hline \multicolumn{4}{|l|}{ Histologic type } \\
\hline Differentiated & 113 & 37.0 & 0.031 \\
\hline Undifferentiated & 57 & 27.0 & \\
\hline \multicolumn{4}{|l|}{ Macroscopic classification } \\
\hline Early & 46 & 68.1 & $<0.001$ \\
\hline Advanced & 124 & 21.4 & \\
\hline \multicolumn{4}{|l|}{ Alarm sign } \\
\hline Absence & 133 & 38.3 & $<0.001$ \\
\hline Presence & 37 & 15.8 & \\
\hline \multicolumn{4}{|l|}{ Concomitant disease } \\
\hline Absence & 88 & 29.4 & 0.344 \\
\hline Presence & 82 & 38.6 & \\
\hline
\end{tabular}

Table 3. Multivariate analysis of the prognosis factors for mortality

\begin{tabular}{lrr} 
Factor & Odds ratio (95\% CI) & p-value \\
Clinical course (conservative observation vs. treatment group) & $2.7(1.7-4.2)$ & $<0.001$ \\
Age ( $\geq 90$ yr vs. $<90$ yr) & $1.0(0.5-1.8)$ & 0.997 \\
Sex (male vs. female) & $1.0(0.7-1.5)$ & 0.941 \\
Histology (undifferentiated vs. differentiated) & $1.4(0.9-2.1)$ & 0.107 \\
Macroscopic classification (advanced vs. early) & $2.4(1.4-4.1)$ & 0.001 \\
Comorbidity (presence vs. absence) & $0.8(0.6-1.2)$ & 0.283 \\
Alarm sign (presence vs. absence) & $1.9(1.2-3.0)$ & 0.009 \\
\hline
\end{tabular}

CI, confidence interval.

lower in the treatment group than in the conservative observation group who refused the recommended treatment, regardless whether early or advanced GC had been diagnosed (Fig. 4). In addition, the survival according was evaluated to treatment modality. The overall survival rate of the ER and surgery group was similar and the disease-specific mortality of the ER and surgery group was also similar (data not shown).

\section{Effects of clinicopathological characteristics on progno- sis}

Seven factors (clinical course, age, sex, histologic type, macroscopic classification, alarm sign, and concomitant disease) were evaluated by univariate analyses to investigate the associations of the clinicopathological characteristics with the prognosis. Significant differences were observed for the clinical course $(p<0.001)$, age $(p=0.027)$, histologic type $(\mathrm{p}=0.031)$, alarm sign $(\mathrm{p}<0.001)$, and macroscopic classification $(\mathrm{p}<0.001)$ (Table 2$)$. The same seven factors were then 
analyzed by multivariate analysis, which revealed the clinical course $(p<0.001)$, alarm sign ( $p=0.009)$, and macroscopic classification $(\mathrm{p}<0.001)$ to be independent prognosis factors (Table 3).

\section{Discussion}

As the society ages, clinicians are increasingly being confronted with the challenges of treating elderly patients. In Korea, the number of patients diagnosed with GC at extremely old ages has been increasing [1]. Several reports have shown that older patients tend to receive less treatment for GCs than younger patients [14-18]. When clinicians make the decision to treat cancer, they consider the cancer stage, the patient's performance status, deterioration of the patient's mental status, and concomitant disease together; they do not rely on the patient's age alone. Generally, the prognosis of elderly patients has been thought to be worse than that of younger patients because of concomitant disease. On the other hand, deaths due to other concomitant diseases amounted to $34 \%-37 \%$ of the total deaths in $>80$-year-old patients with GC [11,19]. Matsushita et al. [20] investigated patients with GC who were older than 80 years and reported that the survival rate after surgical treatment was significantly higher than that for conservative observation, regardless of the patient's performance status and irrespective of whether his or her mental status had deteriorated. The macroscopic classification of the case (e.g., early or advanced cancer) was an important prognostic factor. Therefore, they recommended surgical treatment for patients older than 80 years [20]. Treatments are recommended for GC, even for older patients with age-associated comorbidities that are under control [21]. In a study of the natural history of early GC, Tsukuma et al. [22] reported that the cumulative 5-year risk for progression to an advanced stage was $63.0 \%$. Even patients with early GC usually die within 3 years in the absence of treatment [20]. On the other hand, Katai et al. [23] reported that when a gastrectomy had been performed safely by specialists, the survival rate of elderly patients with early GC was not significantly different from that of the general population. Therefore, the treatment of early GC should not be discouraged in very elderly patients. In this study, patients in the treatment group, those with early GC, and those without an alarm sign showed higher overall survival rates than the others.

Currently, ER is widely accepted as a standard treatment for early GC [24]. Compared to gastrectomy, ER is a less invasive treatment for GC and has many advantages. These advantages include preservation of the stomach, which increases the quality of life and reduces the length of hospitalization compared to gastrectomy. Furthermore, ER-related complications result from the difficulties encountered when performing the resection endoscopically, which are associated with the tumor size and location, rather than age $[6,8]$. Several reports have shown that ER is a safe and feasible treatment for GC in elderly patients [5,6,8]. Etoh et al. [25] reported that the results of an endoscopic mucosal resection in patients older than 80 years were outstanding, and were the same as those obtained via gastrectomy. Therefore, elderly patients with earlier-stage cancer should receive lessinvasive treatments. As the techniques of preoperative and perioperative management have improved, the outcomes of gastric surgery in the elderly have also shown favorable results $[23,26]$. Therefore, treatment should not be discouraged for elderly patients with GC. Similarly, the overall survival rate of the patients in the present study's treatment group was significantly higher than that in the conservatively observed group.

In this study, advanced GC accounted for $72.9 \%$ of cases. The percentage of patients with advanced cancer was significantly higher in the conservative observation group than in the treatment group. Advanced-stage cancers were a cause of the poor prognosis. Therefore, it is important to recognize cancers at the earlier stage to provide a better prognosis in elderly patients. In a previous study, the prevalence of preexisting concomitant disease was significantly higher in elderly patients [8]. In the present study, 88 patients (51.8\%) did not have any concomitant disease. Furthermore, 143 patients (84.1\%) presented with symptoms and 37 patients (21.8\%) showed an alarm sign. In addition, patients with an alarm sign had advanced-stage cancers. Therefore, elderly patients who are being followed up for concomitant disease also need to be checked for gastrointestinal disease. When patients have symptoms, the clinicians need to consider further evaluations.

This study had some limitations. This study was retrospective and could not investigate exactly how the decision was made as to whether to treat the patients or not. In addition, the TNM classification of the patients could not be evaluated because the staging of the patients in the conservative group could not be investigated accurately. In this study, however, the overall survival of the patients with advanced GC was significantly lower than in the early GC. Furthermore, the macroscopic classification was an independent prognosis factor in multivariate analysis. 


\section{Conclusion}

Chronological age alone should not be used as a strategy of determining if treatment will be administered for GC. Patients with early GC or lower-risk preexisting comorbidities should not be discouraged from treatment, even if they are older than 85 years.

\section{Conflicts of Interest}

Conflict of interest relevant to this article was not reported.

\section{References}

1. Jung KW, Won YJ, Kong HJ, Oh CM, Seo HG, Lee JS. Prediction of cancer incidence and mortality in Korea, 2013. Cancer Res Treat. 2013;45:15-21.

2. Lim D, Ha M, Song I. Trends in the leading causes of death in Korea, 1983-2012. J Korean Med Sci. 2014;29:1597-603.

3. Choi KS, Jun JK, Suh M, Park B, Noh DK, Song SH, et al. Effect of endoscopy screening on stage at gastric cancer diagnosis: results of the National Cancer Screening Programme in Korea. Br J Cancer. 2015;112:608-12.

4. Park CH, Lee H, Kim DW, Chung H, Park JC, Shin SK, et al. Clinical safety of endoscopic submucosal dissection compared with surgery in elderly patients with early gastric cancer: a propensity-matched analysis. Gastrointest Endosc. 2014;80: 599-609.

5. Kakushima N, Fujishiro M, Kodashima S, Muraki Y, Tateishi A, Yahagi N, et al. Technical feasibility of endoscopic submucosal dissection for gastric neoplasms in the elderly Japanese population. J Gastroenterol Hepatol. 2007;22:311-4.

6. Isomoto H, Ohnita K, Yamaguchi N, Fukuda E, Ikeda K, Nishiyama H, et al. Clinical outcomes of endoscopic submucosal dissection in elderly patients with early gastric cancer. Eur J Gastroenterol Hepatol. 2010;22:311-7.

7. Ishii N, Uchida S, Itoh T, Horiki N, Matsuda M, Setoyama T, et al. Endoscopic submucosal dissection with a combination of small-caliber-tip transparent hood and flex knife for superficial esophageal neoplasia. Is it safe for elderly patients? Surg Endosc. 2010;24:2110-9.

8. Tokioka S, Umegaki E, Murano M, Takeuchi N, Takeuchi T, Kawakami K, et al. Utility and problems of endoscopic submucosal dissection for early gastric cancer in elderly patients. J Gastroenterol Hepatol. 2012;27 Suppl 3:63-9.

9. Arai T, Esaki Y, Inoshita N, Sawabe M, Kasahara I, Kuroiwa $\mathrm{K}$, et al. Pathologic characteristics of gastric cancer in the elderly: a retrospective study of 994 surgical patients. Gastric Cancer. 2004;7:154-9.

10. Townsley C, Pond GR, Peloza B, Kok J, Naidoo K, Dale D, et al. Analysis of treatment practices for elderly cancer patients in Ontario, Canada. J Clin Oncol. 2005;23:3802-10.

11. Moriguchi S, Maehara Y, Korenaga D, Sugimachi K, Nose Y. Relationship between age and the time of surgery and prognosis after gastrectomy for gastric cancer. J Surg Oncol. 1993;
52:119-23.

12. Takeda J, Tanaka T, Koufuji K, Kodama I, Tsuji Y, Kakegawa $\mathrm{T}$. Gastric cancer surgery in patients aged at least 80 years old. Hepatogastroenterology. 1994;41:516-20.

13. Eguchi T, Fujii M, Takayama T. Mortality for gastric cancer in elderly patients. J Surg Oncol. 2003;84:132-6.

14. Damhuis RA, Tilanus HW. The influence of age on resection rates and postoperative mortality in 2773 patients with gastric cancer. Eur J Cancer. 1995;31A:928-31.

15. Lawrence W Jr, Menck HR, Steele GD Jr, Winchester DP. The National Cancer Data Base report on gastric cancer. Cancer. 1995;75:1734-44.

16. Janes RH Jr, Niederhuber JE, Chmiel JS, Winchester DP, Ocwieja KC, Karnell JH, et al. National patterns of care for pancreatic cancer. Results of a survey by the Commission on Cancer. Ann Surg. 1996;223:261-72.

17. Benhamiche AM, Faivre J, Tazi AM, Couillault C, Villing AL, Rat $P$. Time trends in diagnostic strategy, treatment, and prognosis of gastric cancer in the elderly: a population based study. Eur J Cancer Prev. 1997;6:71-7.

18. Roviello F, Marrelli D, De Stefano A, Messano A, Pinto E, Carli A. Complications after surgery for gastric cancer in patients aged 80 years and over. Jpn J Clin Oncol. 1998;28:116-22.

19. Kitamura K, Yamaguchi T, Taniguchi H, Hagiwara A, Yamane $\mathrm{T}$, Sawai K, et al. Clinicopathological characteristics of gastric cancer in the elderly. Br J Cancer. 1996;73:798-802.

20. Matsushita I, Hanai H, Kajimura M, Tamakoshi K, Nakajima T, Matsubayashi Y, et al. Should gastric cancer patients more than 80 years of age undergo surgery? Comparison with patients not treated surgically concerning prognosis and quality of life. J Clin Gastroenterol. 2002;35:29-34.

21. Lambert R. Treatment of early gastric cancer in the elderly: leave it, cut out, peel out? Gastrointest Endosc. 2005;62:872-4.

22. Tsukuma H, Oshima A, Narahara H, Morii T. Natural history of early gastric cancer: a non-concurrent, long term, follow up study. Gut. 2000;47:618-21.

23. Katai H, Sasako M, Sano T, Fukagawa T. Gastric cancer surgery in the elderly without operative mortality. Surg Oncol. 2004;13:235-8.

24. Soetikno R, Kaltenbach T, Yeh R, Gotoda T. Endoscopic mucosal resection for early cancers of the upper gastrointesti- 
nal tract. J Clin Oncol. 2005;23:4490-8.

25. Etoh T, Katai H, Fukagawa T, Sano T, Oda I, Gotoda T, et al. Treatment of early gastric cancer in the elderly patient: results of EMR and gastrectomy at a national referral center in Japan.
Gastrointest Endosc. 2005;62:868-71.

26. Coniglio A, Tiberio GA, Busti M, Gaverini G, Baiocchi L, Piardi T, et al. Surgical treatment for gastric carcinoma in the elderly. J Surg Oncol. 2004;88:201-5. 